\title{
The Head-Fake Effect in Basketball Is Based on the Processing of Head Orientation, but Not on Gaze Direction
}

\author{
Matthias Weigelt* ${ }^{\circledR}$, Iris Güldenpenning, Yvonne Steggemann-Weinrich \\ Department of Sport \& Health, University of Paderborn, Paderborn, Germany \\ Email: *matthias.weigelt@uni-paderborn.de
}

How to cite this paper: Weigelt, M., Güldenpenning, I., \& Steggemann-Weinrich, Y. (2020). The Head-Fake Effect in Basketball Is Based on the Processing of Head Orientation, but Not on Gaze Direction. Psychology, 11, 1493-1510.

https://doi.org/10.4236/psych.2020.1110095

Received: August 31, 2020

Accepted: October 12, 2020

Published: October 15, 2020

Copyright $\odot 2020$ by author(s) and Scientific Research Publishing Inc. This work is licensed under the Creative Commons Attribution International License (CC BY 4.0).

http://creativecommons.org/licenses/by/4.0/

\begin{abstract}
The head fake in basketball has been used as a paradigmatic example to investigate the cognitive mechanisms of deceptive actions in sports. When a player looks to one side while passing the ball to the opposite side, responses of the observer are slower and more error prone than when the player looks into the pass direction. It is not known if this head-fake effect is based on the processing of the action-irrelevant gaze direction or on the head orientation, or on both. Three experiments were designed to examine the impact of these two different sources of information conflict during the processing of head fakes in basketball. In Experiment 1, the size of the head-fake effect was not reduced when the eyes of the player were covered. In Experiment $2 a$ and $2 b$, the relative influence of gaze direction and head orientation was disentangled. In both experiments, the head-fake effect remained fully present when only the head orientation was changed while the eyes gazed into the pass direction, whereas (vice versa) the effect was (almost) gone when the eyes looked away while the head was oriented into the pass direction. These findings suggest that the head-fake effect in basketball is based on the automating processing of the head orientation, but not on the (otherwise socially important) gaze information.
\end{abstract}

\section{Keywords}

Deceptive Action, Conflict Processing, Head Fake, Perception in Sports

\section{Introduction}

Deliberate deception is common practice in game sports and martial arts (Güldenpenning, Kunde, \& Weigelt, 2017). The intention behind the use of deceptive actions is to gain a benefit over the opponent by either hiding action-relevant 
movement cues for as long as possible or to provide misleading cues in order to provoke an incorrect response in the observer (Jackson, Warren, \& Abernethy 2006). An example for the former strategy is the poke shot in volleyball, where the initial movement phase is similar to the volleyball smash, and only in the final phase, the ball is being softly "poked" across the net and to the opponent's side (Güldenpenning, Steinke, Koester, \& Schack, 2013). This leads to a delayed recognition of the fake attack (i.e., the poke shot), upon which the opponent either does not respond at all, responds too late, or selects a false response. An example for the latter strategy is the head fake in basketball, where a player passes to one side, while simultaneously looking into the other direction (Kunde, Skirde, \& Weigelt, 2011). Experimental studies on the head fake in basketball show that reaction times are slower and more error prone to head fakes than to direct passes, a pattern, which signifies the head-fake effect (e.g., Alhaj Ahmad Alaboud, Güldenpenning, Steggemann-Weinrich, Kunde, \& Weigelt, 2016; Güldenpenning, Schütz, Weigelt, \& Kunde, 2020b; Kunde et al., 2011; Weigelt, Güldenpenning, Steggemann-Weinrich, Alhaj Ahmad Alaboud, \& Kunde, 2017). The head fake in basketball, as a paradigmatic example of conflict processing in sport, will be the focus of interest for the remainder of the article.

In a series of studies conducted in our laboratory, we were able to demonstrate that the head-fake effect is rather robust against several manipulations. For example, it is robust against a larger amount of practice. That is, participants still show the effect after more than 2.000 trials (Güldenpenning, Schütz et al., 2020b). Five days of motor training (participants practiced the head-fake action, while another person was observing vis-a-vis) or visual training (participants observed the other person performing the head fake vis-a-vis) were not effective to reduce the effect (Güldenpenning, Kunde, \& Weigelt, Under Review). The deception is even effective in high-level basketball experts, who practiced their sport for many years (Weigelt et al., 2017). Although, experts, but not novices, are able to adapt their responses whenever the head fake is repeated. The robustness of the head-fake effect is also supported by a recent study showing that the effect is still present with different instructions (Güldenpenning, Weigelt, \& Kunde, 2019). Together, the use of a head fake in basketball appears to be a good strategy to deceive the opponent about one's own action intention.

Some manipulations, however, affect the size of the head-fake effect (while the general effect is still present). When the working memory of participants is taxed by another task in a dual-task scenario (e.g., counting backwards by three), the effect is reduced (by 20\%) (Güldenpenning, Kunde, \& Weigelt, 2020a). On the contrary, it is greater, when video sequences of the action are presented, instead of static images (Alhaj Ahmad Alaboud et al., 2016). It can also be modulated by brain stimulation. Here, a recent study demonstrated that anodal transcranial direct current stimulation (tDCS) over the dorsolateral prefrontal cortex (dLPC) - a brain area responsible for conflict adaptation and resolution-but not catodal stimulation, increases the resistance to head fakes in basketball, which is signified by a smaller head-fake effect (Friehs, Güldenpenning, Frings, \& Weigelt, 
2019). Given this overwhelming evidence for the effectiveness of the head-fake effect in basketball, it is fair to say that the deception cannot be ignored by the observer, but rather seems to be processed automatically (although, the size of the effect can be altered by a number of factors). The size of the head-fake effect also varies according to individual differences of the observer's attentional capabilities (Güldenpenning, Weigelt, Memmert, \& Klatt, in press). Observers with higher attentional capabilities are better able to focus their attention on the relevant action feature (pass direction), when judging a basketball head fake, than observers with lower attentional capabilities, as measured with the attention window task (cf. Hüttermann, Memmert, Simons, \& Bock, 2013). The strongest moderator of the size of the effect, however, seems to be the frequency with which the head fake is being used. A frequent presentation of the head fake results in a longer delay of the responses and more erroneous reactions, whereas when the fake occurs very often, the effect is smaller or (sometimes) participants even react faster (Alhaj Ahmad Alaboud, Steggemann, Klein-Soetebier, Kunde, \& Weigelt, 2012; Güldenpenning, Alhaj Ahmad Alaboud, Kunde, \& Weigelt, 2018).

A noticeable detail of all former studies investigating the head fake in basketball is that during the deceptive action the athlete changes not only his/her head orientation, but also the eye-gaze direction. That is, head and eyes are directed opposite to the pass direction. Obviously, this is an experimental confound, which needs to be solved, because it cannot be decided if the head fake is based on the processing of the (otherwise task-irrelevant) head orientation or eye-gaze direction. In this context, it is interesting to note that the deceptive action is termed "head fake" in the English language, but "gaze deception" ("Blicktäuschung") in the German language. Thus, to disentangle the relative impact of head orientation and eye-gaze direction on the size of the head-fake effect in basketball is the aim of the present series of experiments. The results of the present study will also be informative for other previous studies on deceptive actions in sports (e.g., rugby [Jackson et al., 2006; Mori \& Shimada, 2013], soccer [Smeeton \& Williams, 2012], or team handball [Cañal-Bruland \& Schmidt, 2009; Cañal-Bruland, van der Kamp, \& van Kesteren, 2010]), because as far as can be told from this research, head orientation and eye-gaze direction have not been systematically manipulated before.

There is much research on the important role of the eyes in social interaction (e.g., Emery, 2000; Kendon, 1967; Tomasello, 2008). Here, the eyes not only have a perceiving function in the looker, but they serve as a strong social signal, informing the observer about the looker's action intention (cf. Csibra \& Gergely, 2009). In fact, a sudden shift of eye-gaze direction (through overt movements of the eyes), automatically captures the attention of the observer (so called eye-contact effect, e.g., Senju \& Hasegawa, 2005; Senju \& Johnson, 2009; or stare-in-thecrowd effect, von Grünau \& Anston, 1995) and triggers a visual-spatial orientation reflex (e.g., Driver, Davis, Ricciardelli, Kidd, Maxwell, \& Baron-Cohen, 1999; Friesen \& Kingstone, 1998). That is, the observer shifts his/her own focus 
of attention into the direction of the gaze shift of the looker. In naturalistic social interactions, these gaze capture effects arise while eye movements and the head movement co-occur (temporal and spatial overlap), resulting in the so called sudden direct gaze effect (Böckler, van der Wel, \& Welsh, 2014). Thus, eye-gaze direction, eye motion, and head motion can serve as independent cues for attention capture. Although a recent study suggests that eye gaze and eye motion seem to be responsible for the sudden direct gaze effect, but not head motion (van der Wel, Welsh, \& Böckler, 2018).

Langton and colleagues (Langton, 2000; Langton \& Bruce, 1999) investigated the relative impact of eye-gaze direction and head orientation on participant's response selection in several studies, which are most relevant to the present series of experiments. In a Stroop-like task, Langton (2000) presented pictures of a human face in a way that the head's orientation and eye-gaze direction were either upwards, downwards, left, or right, resulting in congruent or incongruent head-orientation vs. gaze-direction combinations. That is, in one half of the pictures, eye-gaze direction and head orientation were the same (i.e., congruent conditions e.g., eyes gaze to the right and head orientation to the right), whereas in the other half, they were placed into conflict (i.e., incongruent; e.g., eyes gaze to the left and head oriented to the left). Participants were asked to classify the eye-gaze direction or the head orientation in separate experimental blocks. For both tasks, responses were slower under conditions in which the task-irrelevant cue conflicted with the task-relevant cue. The effects proofed to be independent, showing that eye-gaze direction and head orientation seemed to be processed automatically and represent valid cues for attention capture (Experiments 1 and 2). Importantly, head orientation and eye-gaze direction also produced independent congruency effects when both cues were task irrelevant (i.e., participants had to respond to the spoken words "up", “down", "left", "right"; Experiment 3).

From the previous studies using static images (e.g., Kunde et al., 2011; Weigelt et al., 2017) or using dynamic videos (e.g., Güldenpenning et al., 2020a, 2020b, 2018), the question if the head-fake effect in basketball is based on the processing of the action-irrelevant eye-gaze direction or on the head orientation, or on both, cannot be answered, because the head orientation and eye-gaze direction were always confounded (i.e., head and gaze were always oriented into the same direction). Previous studies on social attention examined the relative impact of eye-gaze direction and head orientation and demonstrated that both cues are automatically processed and represent informative cues for attention capture (e.g., Böckler et al., 2014; Langton, 2000; Langton \& Bruce, 1999). Three experiments were designed to disentangle the relative impact of these two different sources of information conflict during the processing of head fakes in basketball.

\section{Experiment 1}

Experiment 1 examines the head-fake effect when the eyes are not visible. There- 
fore, the size of the head-fake effect for stimulus material of a basketball player with the eyes being visible was compared to the size of the head-fake effect with the eyes being occluded. The following predictions were made: (1) If the head-fake effect is only based on eye-gaze direction, then the head-fake effect should only occur, when the eyes are visible, but be eliminated when the eyes are occluded. (2) If the head-fake effect is solely based on head orientation, then the head-fake effect should occur in the same size for visible and for occluded eyes. (3) If head orientation and eye-gaze direction are (both) independently sufficient to deceive the observer, head orientation alone (i.e., when the eyes are occluded) should also interfere with pass direction and provoke a head-fake effect, which is presumably somewhat smaller than the head-fake effect for visible eyes. (4) If head orientation and eye-gaze direction were only sufficient to conjointly deceive the observer, the head-fake effect should be absent for the stimuli with occluded eyes.

\subsection{Methods}

\subsubsection{Participants}

Twenty-four students enrolled in the sport science program were recruited as participants ( 14 females, mean age $=21.0$ years; $S D=2.1$ years). They had no specific experience in basketball. All participants reported normal or corrected-to-normal vision and had no knowledge of the expected outcome of this experiment. Each participant gave informed consent to participate. They were not paid for participation and did not receive any other reward, such as course credits. The single experimental session lasted about 30 minutes. All rights of the participants were protected, and the experiment was carried out according to the 1964 Declaration of Helsinki. Approval for the study was provided by the local ethics committee.

\subsubsection{Apparatus and Stimuli}

Photographic pictures (i.e., portraits) of a male basketball player with a ball in his hands positioned at chest height were used in the present study. The basketball player was recorded whilst imitating to pass the ball to the left or to the right side, either with or without performing a head fake. These pictures were also used with the basketball player having occluded eyes (see Figure 1). All stimuli were presented in color on a black background of a 22 -inch LCD monitor, with a frame rate of $60 \mathrm{~Hz}$. The presentation of the stimulus material was controlled with an IBM-compatible personal computer and the software Presentation (Version 14.5, http://www.neurobs.com). Responses to the stimuli were single key presses on a standard computer keyboard with a polling rate of $1000 \mathrm{~Hz}$ (i.e., timing accuracy of $1 \mathrm{~ms}$ ). Key presses were carried out with the index fingers of each hand, with the "."-key (for passes to the right side) and the " $\mathrm{X}$ "-key (for passes to the left side). This experiment, as well as Experiments $2 \mathrm{a}$ and $2 \mathrm{~b}$ was carried out in a darkened room without windows.

\subsubsection{Procedure and Design}

Participants were given written instructions to respond to the pass direction as 
direct pass

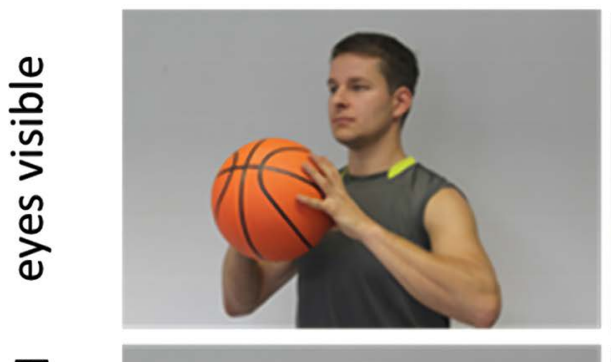

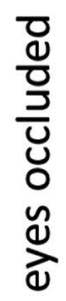

head fake
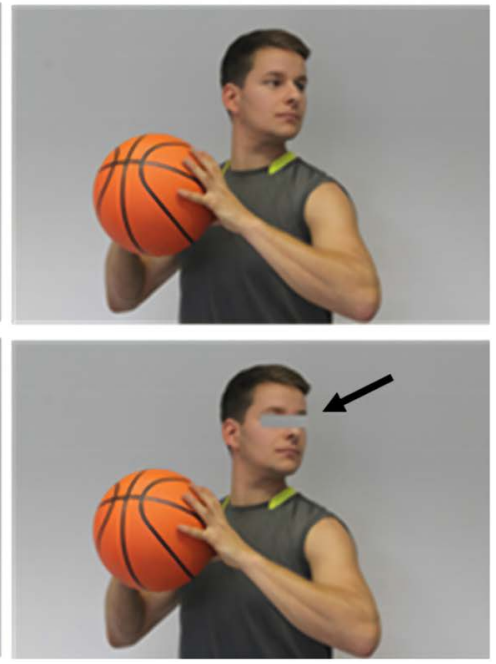

Figure 1. Stimulus material used in Experiment 1. Displayed are directs passes (left column) and head fakes (right column) with the eyes being visible (upper row) or being occluded (lower row). Please note, the black arrow is only shown for illustration purposes here and was not used in the experiment. Stimuli for passes to the right were mirror images of the stimuli displayed here.

quickly and accurately as possible. The first block of 16 trials was considered as practice to familiarize participants with the experiment. During this time, the investigator stayed in the room to answer any questions. Data from this block were not evaluated. This practice block was followed by two different test blocks of 160 trials each, which were separated by a short break, if participants wanted to rest. In one block of these two blocks, the non-occluded stimuli were used (i.e., eyes being visible), whereas in the other block, the occluded stimuli were used (i.e., eyes being occluded/not visible). Within each block, direct passes and head fakes occurred randomized, and equally often (i.e., 50\%). The order of experimental blocks (non-occluded vs. occluded) was balanced between participants.

Each trial began with the presentation of a central fixation cross $(250 \mathrm{~ms})$, which was followed by the target picture. The target picture remained on the screen until a response was given. After the trial ended, participants received feedback about their answer. If there was an error, the word "Fehler" (German for "error") appeared on the screen for $500 \mathrm{~ms}$, followed by a $500 \mathrm{~ms}$ blank screen. If the answer was correct, a blank screen appeared for $1000 \mathrm{~ms}$ before the next trial began.

\subsection{Results}

RTs were analyzed for correct responses and error rates for incorrect responses $(0.9 \%)$. Also, $0.2 \%$ of the data was excluded from further analyses, because RTs were either below $100 \mathrm{~ms}$ (considered as anticipations) or higher than $1000 \mathrm{~ms}$ (considered as outliers). 


\subsubsection{Reaction Times (RT)}

RTs are illustrated in Figure 2 (line graph). The mean RTs were submitted to a two-way ANOVA with the factors action (direct pass vs. head fake) and occlusion (eyes visible vs. eyes occluded) as repeated measures. The analysis of RTs revealed a significant main effect for the factor action $[F(1,23)=23.702, p<$ $\left.0.001 ; \eta_{p}^{2}=0.51\right]$, pointing out that participants responded faster to direct passes ( $350 \mathrm{~ms}, S E=7.7 \mathrm{~ms}$ ) than to head fakes $(357 \mathrm{~ms}, S E=7.3 \mathrm{~ms}$ ). The main effect for occlusion and the interaction between the factors action and occlusion did not reach significance (both $p s>0.10$ ).

\subsubsection{Error Rates (ER)}

ERs are illustrated in Figure 2 (bar graph). The mean ERs were submitted to a two-way ANOVA with the factors action (direct pass vs. head fake) and occlusion (eye visible vs. eye occluded) as repeated measures. Although descriptively participants performed more errors in head-fake trials $(1.1 \%, S E=0.2 \%)$, as compared to trails with direct passes $(0.7 \%, S E=0.3 \%)$, there were no significant main effects for action and for occlusion, as well as no significant interaction of these two factors (all $p s>0.10$ ).

\subsection{Discussion Experiment 1}

The analysis revealed that the head-fake effect was present under both occlusion conditions, that is, when the eyes were visible and when they were occluded, respectively. Moreover, the head-fake effect was of equal size for occluded and visible eyes, which is in line with our second prediction, namely that the head-fake effect is solely based on head orientation. This finding is surprising considering

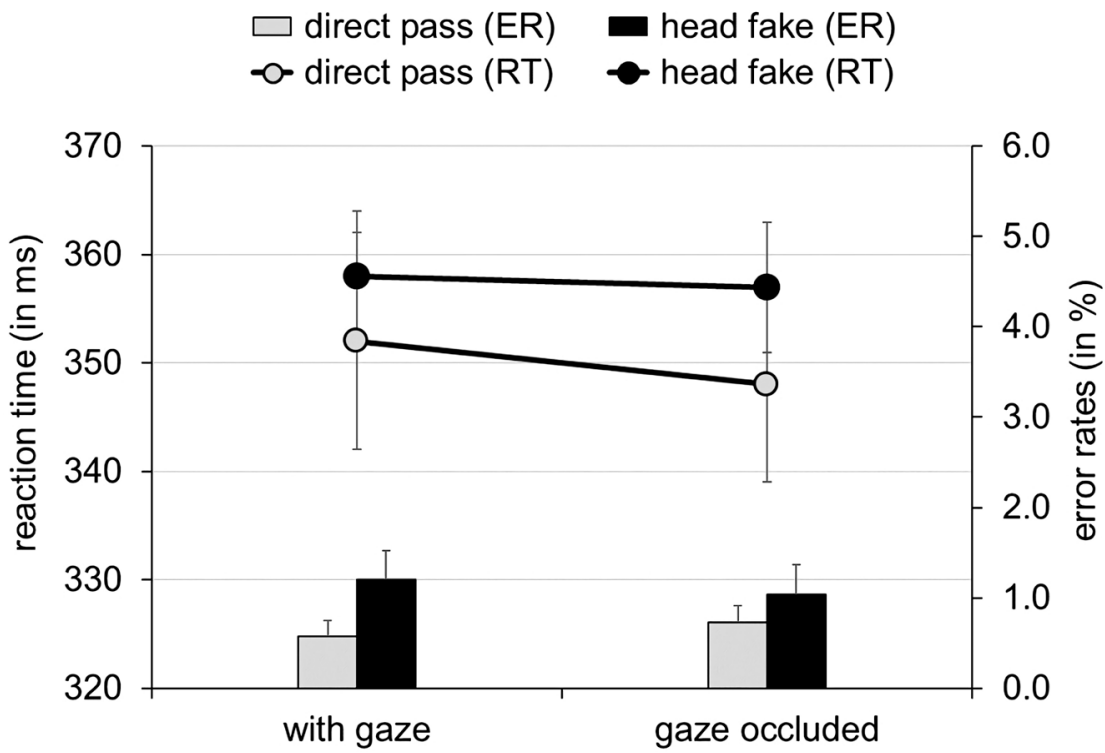

Figure 2. The solid lines illustrate reaction times ( \pm standard errors of means) for direct passes (light grey circles) and head fakes (black circles) in Experiment 1. The bars illustrate the corresponding error rates ( \pm standard errors of means) for direct passes (light grey bars) and head fakes (black bars). 


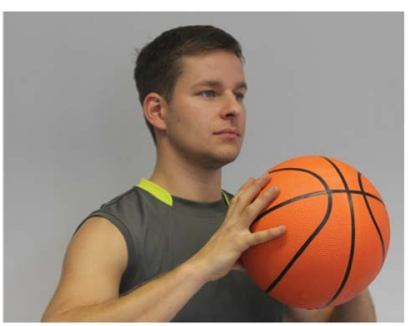

(A)

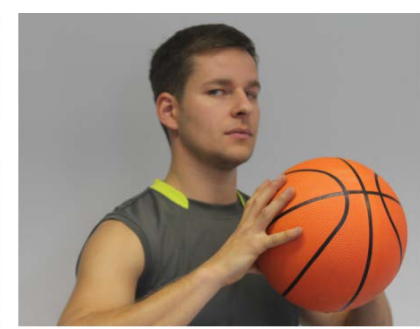

(B)

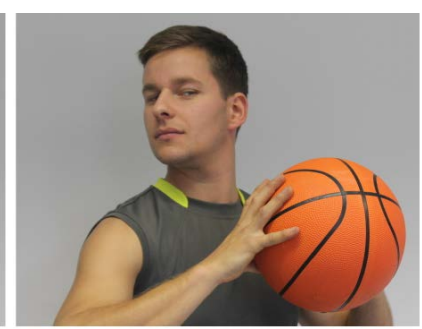

(C)

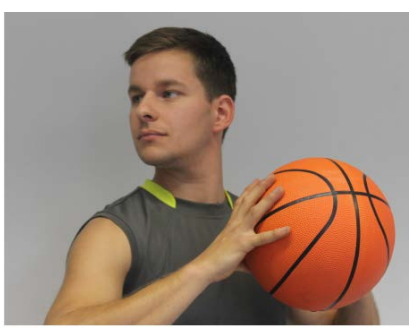

(D)

Figure 3. Depicted are the stimuli used for passes to the right under the different conditions in Experiment 2a (and Experiment 2b): (A) Eye-gaze direction and head orientation are congruent to the pass direction (direct pass; i.e., all congruent), (B) head orientation is congruent, but eye-gaze direction is incongruent to the pass direction (gaze fake; i.e., gaze incongruent), (C) eye-gaze direction is congruent, but head orientation is incongruent to the pass direction (head fake; i.e., head incongruent), and (D) eye-gaze direction and head orientation are incongruent to the past direction (head and gaze fake; i.e., head and gaze incongruent). Stimuli for passes to the left were mirror images of the stimuli displayed here. 
the effect, then the manipulation of only one of the cues should result in a smaller head-fake effect than manipulating both cues. If the latter were true, then it would be of further interest to which extent each of the two cues contribute to the effect.

\subsection{Methods}

\subsubsection{Participants}

Thirty-six new sport science students were recruited as participants (20 females, mean age $=22.5$ years; $S D=3.18$ years). They had no specific experience in basketball. All participants reported normal or corrected-to-normal vision and had no knowledge of the expected outcome of the experiment. Each participant gave informed consent to participate. They were not paid for participation and did not receive any other reward, such as course credits. The single experimental session lasted about 30 minutes. All rights of the participants were protected, in accordance with the ethical standards of the 1964 Declaration of Helsinki. The study was approved by the university's local ethics committee.

\subsubsection{Apparatus and Stimuli}

The design of the stimuli was directly inspired by the pictures used in the experiments of Langton (2000). In this way, they represent a basketball version of the manipulations undertaken by this author. To this end, photographic pictures (i.e., portraits) of a male basketball player with a ball in his hands positioned at chest height were used in the present study. The basketball player was recorded whilst imitating to pass the ball to the left or to the right side. Four different types of stimulus material were used in the experiment (see Figure 3), namely pictures of passes depicting the basketball player (A) with eye-gaze direction and head orientation both congruent to the pass direction (direct pass; i.e., all congruent), (B) with head orientation congruent, but eye-gaze direction incongruent to pass direction (gaze fake; i.e., gaze incongruent), (C) with eye-gaze direction congruent, but head orientation incongruent to pass direction (head fake; i.e., head incongruent), (D) with eye-gaze direction and head orientation both incongruent to pass direction (head and gaze fake; i.e., head and gaze incongruent). All stimuli were presented in color on a black background of a 22 -inch LCD monitor, with a frame rate of $60 \mathrm{~Hz}$. The presentation of the stimulus material was controlled with an IBM-compatible personal computer and the software Presentation (Version 14.5, http://www.neurobs.com). Responses to the stimuli were single key presses on a standard computer keyboard with a polling rate of $1000 \mathrm{~Hz}$ (i.e., timing accuracy of $1 \mathrm{~ms}$ ). Key presses were carried out with the index fingers of each hand, with the "."-key (for passes to the right side) and the "X"-key (for passes to the left side).

\subsubsection{Procedure and Design}

Participants were given written instructions to respond to the pass direction as quickly and accurately as possible. The first block of 16 trials was considered as 
practice to familiarize participants with the experiment. During this time, the investigator stayed in the room to answer any questions. Data from this block were not evaluated. This practice block was followed by three different test blocks of 120 trials each, which were separated by short breaks if participants wanted to rest. The three test blocks differed with regard to the incongruent stimulus material. That is, each test block consisted of 50\% congruent stimuli A (cf. Figure 3), but the incongruent stimuli varied across the three test blocks ( $50 \%$ incongruent stimuli from either $\mathrm{B}$, or $\mathrm{C}$, or $\mathrm{D}$ ). This resulted in a gaze-fake block (i.e., all congruent vs. gaze incongruent), a head-fake block (i.e., all congruent vs. gaze incongruent), and a head- \& gaze-fake block (i.e., all congruent vs. head and gaze incongruent). The order of the test blocks was randomized across participants. Each trial began with the presentation of a central fixation cross. After a fixed time-interval of $250 \mathrm{~ms}$, the stimulus picture was presented and remained on the screen until a response was given. After the trial ended, participants received feedback about their answer. If there was an error, the word "Fehler" (German for "error") appeared on the screen for $500 \mathrm{~ms}$. If the answer was correct, the next trial began immediately with the appearance of the fixation cross.

\subsection{Results}

RTs were analyzed for correct responses and error rates for incorrect responses (1.4\%). Also, $0.8 \%$ of the data was excluded from further analyses, because RTs were either below $100 \mathrm{~ms}$ (considered as anticipations) or higher than $1000 \mathrm{~ms}$ (considered as outliers).

\subsubsection{Reaction Times (RT)}

RTs are illustrated in Figure 4 (line graph). The mean RTs were submitted to a two-way ANOVA with the factors action (direct pass vs. fake action) and test block (gaze-fake block vs. head-fake block vs. head- \& gaze-fake block) as repeated measures. The analysis of RTs revealed a significant main effect for the factor action $\left[F(1,35)=40.852, p<0.001 ; \eta_{p}^{2}=0.54\right]$, pointing out that participants responded faster to direct passes $(343 \mathrm{~ms}, S E=5.3 \mathrm{~ms})$ than to head fakes (349 ms, $S E=5.7 \mathrm{~ms})$. The main effect for test block also reached significance $\left[F(2,70)=4.179, p<0.001 ; \eta_{p}^{2}=0.11\right]$, as RTs in the gaze-fake block (342 $\mathrm{ms}, S E=5.8 \mathrm{~ms}$ ) were significantly faster than in the head-fake block (349 $\mathrm{ms}$, $S E=5.7 \mathrm{~ms})([t(35)=2.500, p<0.05, d=0.18]$ and in the head- \& gaze-fake block (348 ms, $S E=5.5 \mathrm{~ms}$ ) [ $t(35)=2.188, p<0.05, d=0.18]$. The interaction between action and test block only approached significance $[F(1.573,55.064)=$ $3.105, p=0.064 ; \eta_{p}^{2}=0.08$ ], even though numerically, the gaze-fake effect (3.5 $\mathrm{ms}, S E=1.9 \mathrm{~ms})$ was considerably smaller than the head- \& gaze-fake effect (9.3 $\mathrm{ms}, S E=1.9 \mathrm{~ms})$ and the head-fake effect $(7.2 \mathrm{~ms}, S E=1.4 \mathrm{~ms})$.

\subsubsection{Error Rates (ER)}

Figure 4 (bar graph) shows the mean ER values for the different conditions. The 


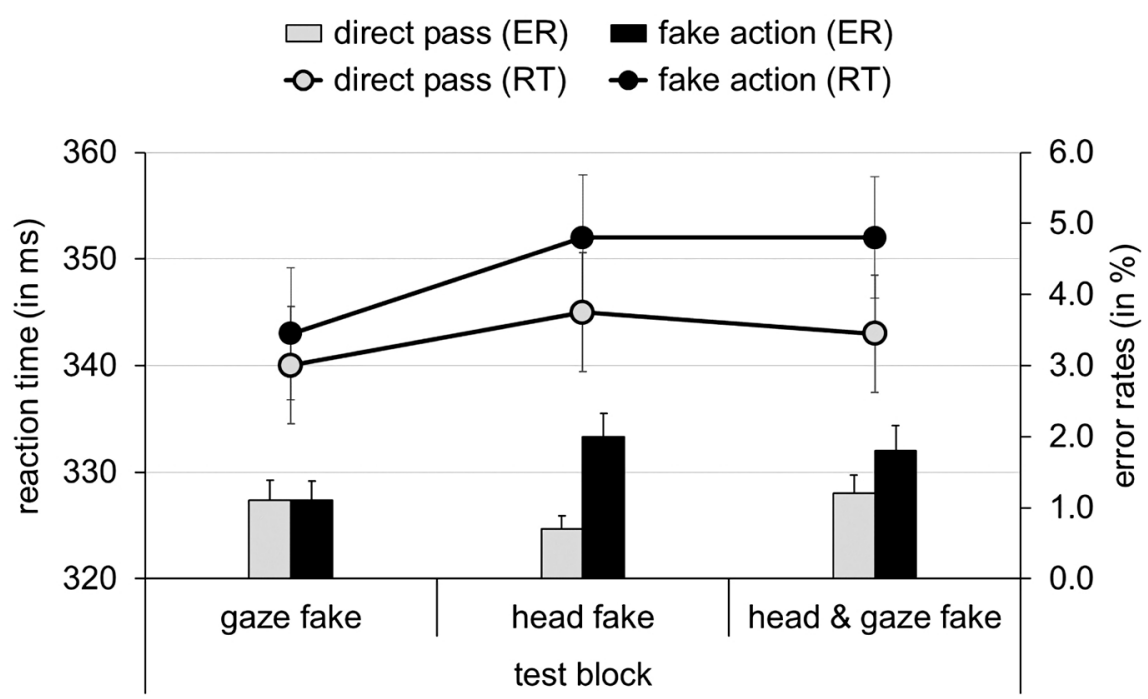

Figure 4. Shows the results for RTs (line graph, \pm standard errors of means) for direct passes (light grey circles) and fake actions (black circles), as a function of test block (gaze-fake block vs. head-fake block vs. head- \& gaze-fake block) in Experiment 2a. The bars illustrate the corresponding error rates ( \pm standard errors of means) for direct passes (light grey bars) and fake actions (black bars).

mean ERs were submitted to a two-way ANOVA with the factors action (direct pass vs. fake action) and test block (gaze-fake block vs. head-fake block vs. head\& gaze-fake block) as repeated measures. The analysis of ERs revealed a significant main effect for the factor action $\left[F(1,35)=10.212, p<0.01 ; \eta_{p}^{2}=0.23\right]$, pointing out that participants performed more errors to fake actions $(1.6 \%, S E=$ $0.3 \%)$ than to direct passes $(1.0 \%, S E=0.2 \%)$. The main effect for test block did not reach significance $(p>0.10)$. The interaction between action and test block only approached significance $\left[F(2,70)=2.562, p=0.084 ; \eta_{p}^{2}=0.07\right]$. Numerically, however, there was no gaze-fake effect (0\%) on ER, while the head-fake effect was $1.3 \%$ and the head- and gaze-fake effect was $0.6 \%$ on the ER.

\subsection{Discussion Experiment 2a}

The results of Experiment 2a are not as straight forward. When the influence of the eye-gaze direction and the head orientation was disentangled by varying the stimulus material, the fake effect was statistically present in RTs and ERs, irrespective of the experimental manipulation. In contrast to Experiment 1, this result suggests that the task-irrelevant eye-gaze direction alone, the head orientation alone, and a combination of eye-gaze direction and head orientation are able to produce a fake effect of equal size. Numerically, however, the effect of eye-gaze direction was considerably smaller $(3.5 \mathrm{~ms}$ and $0 \%)$ than the effect of head orientation alone (7.2 ms and 1.3\%) and the combined effect of head and gaze orientation (9.3 ms and $0.6 \%$ ) for RT and ER, respectively, although the interaction between action and test block failed to reach significance only marginally. It is therefore not clear if eye-gaze direction alone can reliably produce a fake effect (Prediction 1), does not reliably produce a fake effect (Prediction 2), 
or if eye-gaze direction contributes to the fake effect at all (Prediction 3).

\section{Experiment $2 b$}

A detail of the task procedure of Experiment 2a was that the incongruent conditions were present in a blocked design. This led to the situation that trials displaying direct passes, in which the pass direction, eye-gaze direction, and head orientation were all congruent, were presented three times as often as trials for each of the incongruent conditions across the experiment. To control for this overrepresentation of congruent cues, all conditions were presented randomly (i.e., $25 \%$ of the trials for each condition) in Experiment $2 \mathrm{~b}$. The same predictions were made as in Experiment 2a.

\subsection{Methods}

\subsubsection{Participants}

Twenty-four new sport science students were recruited as participants (12 females, mean age $=24.5$ years; $S D=2.28$ years). They had no specific experience in basketball. All participants reported normal or corrected-to-normal vision and had no knowledge of the expected outcome of this experiment. Each participant gave informed consent to participate. They were not paid for participation and did not receive any other reward, such as course credits. The single experimental session lasted about 30 minutes. All rights of the participants were protected, in accordance with the ethical standards of the 1964 Declaration of Helsinki. The study was approved by the local ethics committee of the university.

\subsubsection{Apparatus and Stimuli}

The apparatus and stimulus material were identical to Experiment 2a. However, the four different types of stimulus material (cf. Figure 3) were randomly presented in Experiment 2b, and each type of stimulus occurred for $25 \%$ within the experiment.

\subsubsection{Procedure and Design}

Participants were given written instructions to respond to the pass direction as quickly and accurately as possible. The first block of 16 trials was considered as practice to familiarize participants with the experiment. During this time, the investigator stayed in the room to answer any questions. Data from this block were not evaluated. This practice block was followed by two identical test blocks of 160 trials each, which were separated by short breaks if participants wanted to rest. The four types of stimuli (i.e., A, B, C, D; cf. Figure 3) occurred randomized and equally often (i.e., 25\%) within each test block. Each trial began with the presentation of a central fixation cross. After a fixed time-interval of $250 \mathrm{~ms}$, the stimulus picture was presented and remained on the screen until a response was given. After the trial ended, participants received feedback about their answer. If there was an error, the word "Fehler" (German for "error") appeared on the screen for $500 \mathrm{~ms}$. If the answer was correct, the next trial began immediately 
with the appearance of the fixation cross.

\subsection{Results}

RTs were analyzed for correct responses and error rates for incorrect responses (1.0\%). Also, $0.1 \%$ of the data was excluded from further analyses, because RTs were either below $100 \mathrm{~ms}$ (considered as anticipations) or higher than $1000 \mathrm{~ms}$ (considered as outliers).

\subsubsection{Reaction Times (RT)}

The mean RTs are displayed in Figure 5 (line graph) and were $355 \mathrm{~ms}$ ( $S D=$ $27.4 \mathrm{~ms}$ ) for direct passes (i.e., all congruent), $357 \mathrm{~ms}(S D=29.6 \mathrm{~ms}$ ) for gaze fakes (i.e., gaze incongruent), $363 \mathrm{~ms}(S D=29.7)$ for head fakes (i.e., head incongruent), and $363 \mathrm{~ms}(S D=28.8)$ for head and gaze fakes (i.e., gaze and head incongruent), respectively. The mean RTs were submitted to a one-way ANOVA with the factor condition (all congruent, gaze incongruent, head incongruent, head and gaze incongruent) as repeated measures. The ANOVA yielded a significant main effect for the factor condition $\left[F(1,69)=13.868, p<0.001 ; \eta_{p}^{2}=\right.$ $0.38]$. The differences between the conditions was further analyzed with $t$-test (two-tailed), following Holm's sequential procedure to avoid effects of multiple testing. Significant differences were found for the following comparisons: 1) all congruent vs. head incongruent [ $t(23)=4.797, p<0.001, d=0.28], 2)$ all congruent vs. head and gaze incongruent [ $t(23)=5.485, p<0.001, d=0.29], 3)$ gaze incongruent vs. head incongruent $[t(23)=4.059, p<0.001, d=0.27]$, and 4 ) gaze incongruent vs. head and gaze incongruent $[t(23)=3.661, p=0.001, d=$ 0.29]. The other comparisons did not reach significance.

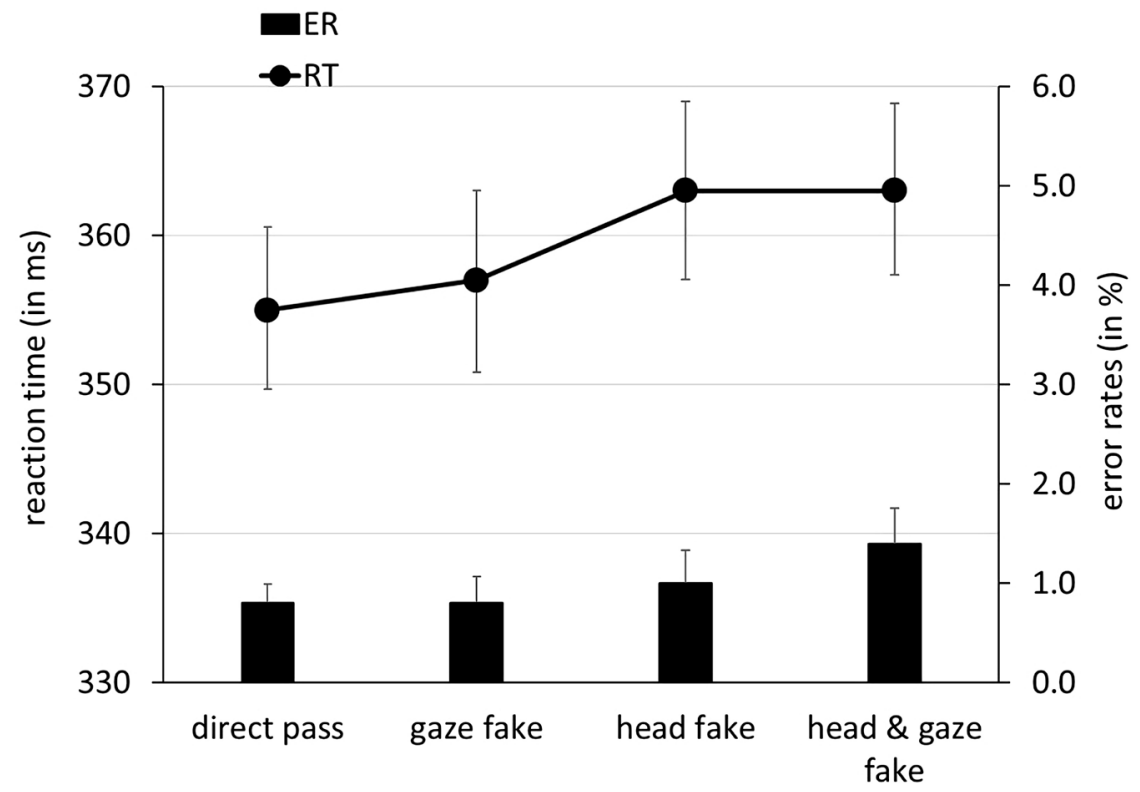

Figure 5. The results for RTs (line graph, \pm standard errors of means) and ERs (bar graph, \pm standard errors of means) for the different stimulus conditions (direct pass, gaze fake, head fake, head and gaze fake) in Experiment $2 b$ are displayed in the figure. 


\subsubsection{Error Rates (ER)}

Figure 5 (bar graph) shows the mean ERs, which were $0.8 \%(S D=1.3 \%)$ for direct passes (i.e., all congruent), $0.8 \%(S D=0.9 \%)$ for gaze fakes (i.e., gaze incongruent), $1.0 \%$ ( $S D=1.3 \%$ ) for head fakes (i.e., head incongruent), and $1.4 \%$ ( $S D$ $=1.1 \%$ ) for head and gaze fakes (i.e., head and gaze incongruent), respectively. The one-way ANOVA for the factor condition was not significant and thus, there was no difference in ER between conditions.

\subsection{Discussion Experiment 2b}

Experiment $2 \mathrm{~b}$ was set up to control for the overrepresentation of congruent cues in Experiment 2a. To this end, all stimulus conditions were presented randomly with the same proportion (i.e., $25 \%$ of the trials for each condition) across the experiment. The pattern of results for the RTs in the present experiment supports Prediction 2, suggesting that the head-fake effect is rather based on the processing of the head orientation, then on the processing of eye-gaze direction. Again, participants responded significantly slower to trials in which the head orientation was incongruent to the pass direction. The effect was significant for the RT data, but not significant in the ER data, although there was a numerical difference pointing into this direction. With an average of $1.0 \%$ response errors across the experiment, participants committed even fewer errors than in the previous two experiments, which possibly prevented small differences to become significant (reminiscent of a floor effect).

\section{General Discussion}

The series of three experiments was set up to examine whether the head-fake effect in basketball is based on the processing of the task-irrelevant eye-gaze direction or the task-irrelevant head orientation. This was warranted, because both types of stimulus information were confounded in the stimuli used in all previous studies on the head fake in basketball (e.g., Kunde et al., 2011; Weigelt et al., 2017). The relative impact of an athlete's gaze direction and head orientation on deceiving an opponent was therefore not known. The results of Experiment 1 and Experiment $2 \mathrm{~b}$ demonstrate that deceiving the opponent with a head fake in basketball is based solely on the automatic processing of the task-irrelevant head orientation. With regard to Experiment 2a, the results were not as clear and an influence of eye-gaze direction on the fake effect seems possible. However, as this effect was numerically small and when considering the pattern of results across all three experiments, it can be argued that the influence of eye-gaze direction on the head-fake effect in basketball is rather dismal. This result is surprising given the many studies attributing a special role to the processing of eye gaze in communication and social interaction (e.g., Emery, 2000; Kendon, 1967; Tomasello, 2008).

From the studies by Langton and colleagues and Böckler et al. (e.g., Böckler et al., 2014; Langton, 2000; Langton \& Bruce, 1999) it could have been assumed that 
eye-gaze direction and head orientation collectively contribute to the head-fake effect. These studies demonstrated that both cues are automatically processed and represent informative cues for attention capture. For the head fake in basketball, however, the otherwise socially relevant eye-gaze direction does not seem to affect the observer, when responding to the pass direction of the opponent. This is somewhat surprising, especially since the stimuli in Experiment 2a and $2 \mathrm{~b}$ were design as closely as possible to the ones used by Langton (2000), who suggested that eye-gaze direction and head orientation are processed as cues independently and defer the responses to the same extent.

We can only speculate why there was no substantial effect of eye-gaze direction in the present series of experiments. First, in the study by Langton (2000) only two cues were manipulated, eye-gaze direction and head orientation, respectively, and one cue was always task-relevant, whereas the other cue was task-irrelevant. In the present Experiments $2 \mathrm{a}$ and $2 \mathrm{~b}$, there were three cues, with the pass direction always being task-relevant, whereas eye-gaze direction and head orientation were both always task-irrelevant. Thus, maybe eye-gaze direction only manifests itself as a conflicting factor when it is the only task-irrelevant cue. Second, in a similar vein, head orientation may be the stronger task-irrelevant cue (as compared to eye gaze orientation) in the basketball situation, capturing all the attention resources, which lead to the distraction. Third, eye-gaze direction is relative to the head, and will thus be slower to process than the head orientation alone. Since the response times were rather small, processing the eye cue might simply be too slow for causing an effect. Fourth, eye-gaze direction might not have taken a meaningful effect, because the way the stimuli were presented did not capture the attention of the observer in the first place (i.e., no eye-contact effect, e.g., Senju \& Hasegawa, 2005; Senju \& Johnson, 2009). That is, only single pictures (and not a series of pictures beginning with an initial picture of a basketball player staring directly at the observer, before the onset of head motion and gaze shift occurred) with averted eye-gaze direction and head orientation were presented. Therefore, it can be argued that presenting an initial picture with the opponent directly facing the observer before the basketball pass would have generated the sudden direct gaze effect (Böckler, van der Wel, \& Welsh, 2014). In fact, a recent study suggested that eye gaze and eye motion seem to be responsible for the sudden direct gaze effect, but not head motion (van der Wel, Welsh, \& Böckler, 2018). Therefore, whether or not direct eye contact before the deceptive action effects the size of the head-fake effect is certainly an intriguing question, which should be considered in a future investigation.

The present results are also useful when considering other previous studies on deceptive actions in sports (e.g., rugby [Jackson et al., 2006; Mori \& Shimada, 2013], soccer [Smeeton \& Williams, 2012], or team handball [Cañal-Bruland \& Schmidt, 2009; Cañal-Bruland et al., 2010]). As far as can be told from this research (especially from the information available on the descriptions of tasks and stimuli in these studies), head orientation and eye-gaze direction have not been 
systematically manipulated before. It appears that during close-up, one-on-one situations in rapidly changing interactive game sports, information on the opponent's passing or movement direction are inferred from rather gross kinematic cues of the body (or body parts) (cf. Brault, Bideau, Kulpa, \& Craig, 2012; Wright, Bishop, Jackson, \& Abernethy, 2011; Wright \& Jackson, 2014), and not from eye-gaze direction. This notion, however, needs to be further investigated for different kinds of deceptive actions in basketball and other interactive team sports.

\section{Conclusion}

The present study disentangled the relative impact on eye-gaze direction and head orientation on the size of the head-fake effect in basketball. From the pattern of results across the series of three experiments, it can be concluded that the head-fake effect is based on the visual processing of (the otherwise task-irrelevant) head orientation information. An incongruent head orientation causes interference with the encoding of the intended pass direction, similar to what has been observed in other conflict paradigms (e.g., Stroop task, Simon task). The processing of the social cue of eye-gaze direction, however, does not affect the size of the head-fake effect in a substantial way.

\section{Conflicts of Interest}

The authors declare no conflicts of interest regarding the publication of this paper.

\section{References}

Alhaj Ahmad Alaboud, M., Güldenpenning, I., Steggemann-Weinrich, Y., Kunde, W., \& Weigelt, M. (2016). Deception in Sports: The Head Fake Effect in Basketball under Quasi-Realistic Conditions. Sportwissenschaft, 46, 223-231.

https://doi.org/10.1007/s12662-016-0401-8

Alhaj Ahmad Alaboud, M., Steggemann, Y., Klein-Soetebier, T., Kunde, W., \& Weigelt, M. (2012). Deception in Sports: An Experimental Study on the Impact of Different Frequency Distributions of Head Fakes in Basketball. Zeitschrift für Sportpsychologie, 19, 110-121. https://doi.org/10.1026/1612-5010/a000075

Böckler, A., van der Wel, R. P., \& Welsh, T. (2014). Catching Eyes: Effects of Social and Non-Social Cues on Attention Capture. Psychological Science, 25, 720-727. https://doi.org/10.1177/0956797613516147

Brault, S., Bideau, B., Kulpa, R., \& Craig, C. M. (2012). Detecting Deception in Movement: The Case of the Side-Step in Rugby. PLoS ONE, 7, e37494. https://doi.org/10.1371/journal.pone.0037494

Cañal-Bruland, R., \& Schmidt, M. (2009). Response Bias in Judging Deceptive Movements. Acta Psychologica, 130, 235-240. https://doi.org/10.1016/j.actpsy.2008.12.009

Cañal-Bruland, R., van der Kamp, J., \& van Kesteren, J. (2010). An Examination of Motor and Perceptual Contributions to the Recognition of Deception from Others' Actions. Human Movement Science, 29, 94-102. https://doi.org/10.1016/j.humov.2009.10.001

Csibra, G., \& Gergely, G. (2009). Natural Pedagogy. Trend in Cognitive Science, 13, 148-153. 
https://doi.org/10.1016/j.tics.2009.01.005

Driver, J., Davis, G., Ricciardelli, P., Kidd, P., Maxwell, E., \& Baron-Cohen, S. (1999). Gaze Perception Triggers Reflexive Visuospatial Orienting. Visual Cognition, 6, 509-540. https://doi.org/10.1080/135062899394920

Emery, N. J. (2000). The Eyes Have It: The Neuroethology, Function and Evolution of Social Gaze. Neuroscience and Biobehavioral Reviews, 24, 581-604. https://doi.org/10.1016/S0149-7634(00)00025-7

Friehs, M. A., Güldenpenning, I., Frings, C., \& Weigelt, M. (2019). Electrify Your Game! Anodal tDCS Increases the Resistance to Head Fakes in Basketball. Journal of Cognitive Enhancement, 4, 62. https://doi.org/10.1007/s41465-019-00133-8

Friesen, C. K., \& Kingstone, A. (1998). The Eyes Have It! Reflexive Orienting Is Triggered by Nonpredictive Gaze. Psychonomic Bulletin \& Review, 5, 490-495. https://doi.org/10.3758/BF03208827

Güldenpenning, I., Alhaj Ahmad Alaboud, M., Kunde, W., \& Weigelt, M. (2018). The Impact of Global and Local Context Information on the Processing of Deceptive Actions in Game Sports: Both Higher Head-Fake Frequencies and Head-Fake Repetitions Reduce the Size of the Head-Fake Effect in Basketball. German Journal of Exercise and Sport Research, 48, 366-375. https://doi.org/10.1007/s12662-018-0493-4

Güldenpenning, I., Kunde, W., \& Weigelt, M. (2017). How to Trick Your Opponent: A Review Article on Deceptive Actions in Interactive Sports. Frontiers in Psychology, 8, Article 917. https://doi.org/10.3389/fpsyg.2017.00917

Güldenpenning, I., Kunde, W., \& Weigelt, M. (2020a). Cognitive Load Reduces Interference by Head Fakes in Basketball. Acta Psychologica, 203, Article ID: 103013. https://doi.org/10.1016/j.actpsy.2020.103013

Güldenpenning, I., Kunde, W., \& Weigelt, M. (Under Review). Effects of Expertise and Motor and Visual Training on the Head-Fake Effect in Basketball.

Güldenpenning, I., Schütz, C., Weigelt, M., \& Kunde, W. (2020b). Is the Head-Fake Effect in Basketball Robust against Practice? Analyses of Trial-by-Trial Adaptations, Frequency Distributions, and Mixture Effects to Evaluate Effects of Practice. Psychological Research, 84, 823-833. https://doi.org/10.1007/s00426-018-1078-4

Güldenpenning, I., Steinke, A., Koester, D., \& Schack, T. (2013). Athletes and Novices Are Differently Capable to Recognize Feint and Non-Feint Actions. Experimental Brain Research, 230, 333-343. https://doi.org/10.1007/s00221-013-3658-2

Güldenpenning, I., Weigelt, M., \& Kunde, W. (2019). Processing Head Fakes in Basketball: Are There Ironic Effects of Instructions on the Head-Fake Effect in Basketball? Human Movement Science, 67, Article ID: 102499.

https://doi.org/10.1016/j.humov.2019.102499

Güldenpenning, I., Weigelt, M., Memmert, D., \& Klatt, S. Processing Deceptive Information in Sports: Individual Differences for Responding to Head Fakes Depends on the Attentional Capability of the Observer. Psychology of Sport and Exercise, in press.

Hüttermann, S., Memmert, D., Simons, D. J., \& Bock, O. (2013). Fixation Strategy Influences the Ability to Focus Attention on Two Spatially Separate Objects. PLoS ONE, 8 , e65673. https://doi.org/10.1371/journal.pone.0065673

Jackson, R. C., Warren, S., \& Abernethy, B. (2006). Anticipation Skill and Susceptibility to Deceptive Movements. Acta Psychologica, 123, 355-371.

https://doi.org/10.1016/j.actpsy.2006.02.002

Kendon, A. (1967). Some Functions of Gaze-Direction in Social Interaction. Acta Psychologica, 26, 22-63. https://doi.org/10.1016/0001-6918(67)90005-4 
Kunde, W., Skirde, S., \& Weigelt, M. (2011). Trust My Face: Cognitive Factors of Head Fakes in Sports. Journal of Experimental Psychology: Applied, 17, 110-127. https://doi.org/10.1037/a0023756

Langton, S. R. H. (2000). The Mutual Influence of Gaze and Head Orientation in the Analysis of Social Attention Direction. The Quarterly Journal of Experimental Psychology: Section A, 53, 825-845. https://doi.org/10.1080/713755908

Langton, S. R. H., \& Bruce, V. (1999). Reflexive Visual Orienting in Response to the Social Attention of Others. Visual Cognition, 6, 541-568. https://doi.org/10.1080/135062899394939

Mori, S., \& Shimada, T. (2013). Expert Anticipation from Deceptive Action. Attention, Perception, \& Psychophysics, 75, 751-770. https://doi.org/10.3758/s13414-013-0435-Z

Senju, A., \& Hasegawa, T. (2005). Direct Gaze Captures Visuospatial Attention. Visual Cognition, 12, 127-144. https://doi.org/10.1080/13506280444000157

Senju, A., \& Johnson, M. H. (2009). The Eye Contact Effect: Mechanism and Development. Trends in Cognitive Science, 13, 127-134.

https://doi.org/10.1016/j.tics.2008.11.009

Smeeton, N. J., \& Williams, A. M. (2012). The Role of Movement Exaggeration in the Anticipation of Deceptive Soccer Penalty Kicks. British Journal of Psychology, 103, 539-555. https://doi.org/10.1111/j.2044-8295.2011.02092.x

Tomasello, M. (2008). Origins of Human Communication. Cambridge, MA: MIT Press. https://doi.org/10.7551/mitpress/7551.001.0001

van der Wel, R. P., Welsh, T., \& Böckler, A. (2018). Talking Heads or Talking Eyes? Effects of Head Orientation and Sudden Onset Gaze Cues on Attention Capture. Attention, Perception, \& Psychophysics, 80, 1-6. https://doi.org/10.3758/s13414-017-1462-y

von Grünau, M., \& Anston, C. (1995). The Detection of Gaze Direction: A Stare-in-theCrowd Effect. Perception, 24, 1297-1313. https://doi.org/10.1068/p241297

Weigelt, M., Güldenpenning, I., Steggemann-Weinrich, Y., Alhaj Ahmad Alaboud, M., \& Kunde, W. (2017). Head Fakes in Sports: Control over the Processing of the Opponent's Gaze Direction in Basketball Experts. Psychonomic Bulletin and Review, 24, 828-834. https://doi.org/10.3758/s13423-016-1140-4

Wright, M. J., \& Jackson, R. C. (2014). Deceptive Body Movements Reverse Spatial Cueing in Soccer. PLoS ONE, 9, e104290. https://doi.org/10.1371/journal.pone.0104290

Wright, M. J., Bishop, D. T., Jackson, R. C., \& Abernethy, B. (2011). Cortical fMRI Activation to Opponents' Body Kinematics in Sport-Related Anticipation: Expert-Novice Differences with Normal and Point-Light Video. Neuroscience Letters, 500, 216-221. https://doi.org/10.1016/j.neulet.2011.06.045 